Website: http://revistas.lamolina.edu.pe/index.php/acu/index

(C) Universidad Nacional Agraria La Molina, Lima - Perú

\title{
Efecto del anillado de ramas, thidiazuron y ácido giberélico en el tamaño del fruto de palto 'Hass' (Persea americana Mill.)
}

\section{Effect of branch girdling, thidiazuron and gibberellic acid on fruit size of 'Hass' avocado (Persea americana Mill.)}

\author{
Sofía Flores $\operatorname{Vivar}^{1} \&$ Jorge Escobedo Alvarez ${ }^{2 *}$ \\ * Autor de correspondencia
}

\section{Resumen}

En tres ensayos sobre árboles de palto 'Hass' de 15 años de edad, se estudió el efecto en el tamaño del fruto de los siguientes tratamientos: anillado de ramas en plena floración y 60 días después de plena floración (ddpf), aplicaciones foliares de thidiazurón (TDZ) $\left(25,50\right.$ y 100 ppm) y aplicaciones foliares de ácido giberélico $\left(\mathrm{AG}_{3}\right)(25$ y 50 ppm). Los reguladores del crecimiento se aplicaron $60 \mathrm{ddpf}$, cuando los frutos tenían $30 \pm 2 \mathrm{~mm}$ de diámetro. Tres semanas antes de la cosecha se midió el diámetro y la longitud de los mismos. El anillado en plena floración no afectó el tamaño de la fruta. Sin embargo, en las ramas anilladas $60 \mathrm{ddpf}$, el diámetro y la longitud se incrementaron en 9,42\% y 13,06 \%, respectivamente, con relación al testigo; además las ramas anilladas no afectaron el tamaño de los frutos de las ramas no anilladas del mismo árbol. Con el TDZ, la longitud de los frutos mejoró en $10,81 \%, 7,38 \%$ y $8,30 \%$ con las tres concentraciones aplicadas $(25,50$ y 100 ppm, respectivamente); pero los diámetros fueron mayores solo con 25 y 50 $\operatorname{ppm}\left(8,11 \%\right.$ y $9,80 \%$ de incremento en comparación con el testigo). Las aplicaciones de $\mathrm{AG}_{3}$ no mejoraron el tamaño de los frutos.

Palabras clave: anillado; thidiazuron; ácido giberélico; plena floración; rama.

\begin{abstract}
In three assays on 'Hass' avocado trees from 15 years of age, the effect on fruit size of the following treatments was studied: branch girdling at full bloom and 60 days after full bloom (dafb), foliar applications of thidiazuron (TDZ) (25, 50 and $100 \mathrm{ppm}$ ) and foliar applications of gibberellic acid $\left(\mathrm{GA}_{3}\right)(25$ and $50 \mathrm{ppm})$. The growth regulators were applied at $60 \mathrm{dafb}$ when the fruits size were $30 \pm 2 \mathrm{~mm}$ in diameter. Three weeks before harvest, the diameter and length of the fruits were measured. Girdling in full bloom did not affect the fruit size. However, on branches girdled 60 dafb, the diameter and length of fruits increased in $9,42 \%$ and $13,06 \%$, respectively compared to the control trees; in addition, girdled branch did not affect the size of the fruits of the not girdled branches of the same tree. With TDZ, the length of the fruit increased $10,81 \% ; 7 ; 38 \%$ and $8,30 \%$ with the three concentrations applied $(25,50$, and 100 ppm, respectively); but the diameters were higher only for 25 and $50 \mathrm{ppm}(8,11 \%$ and 9,80\% increase compared to the control trees). GA 3 applications did not improve the fruit size.
\end{abstract}

Keywords: girdling; thidiazuron; gibberellic acid; full bloom; branch.

\section{Introducción}

Una particularidad genética que puede resultar poco deseable del fruto del palto 'Hass' es su tamaño relativamente pequeño, lo que bajo ciertas condiciones climáticas resulta en la producción de frutas con calibres muy reducidos, que muchas veces están por debajo del promedio, el mismo que Jaque (2006) considera que se encuentra entre 160 y 320 gramos. Los porcentajes de la fruta de bajo valor comercial pueden variar entre $20 \%$ y 60 $\%$, dependiendo de aspectos como clima, carga y edad del árbol, estado sanitario y prácticas culturales (Bergh, 1984; Zilkah \& Klein, 1987; Cutting, 1993; Moore-Gordon et al., 1998). El tamaño final de un fruto de palto está en función del número de células que lo conforman; se considera que una vez cuajado el fruto, la etapa más sensible en la división celular se prolonga hasta 55 a 60 días después. Por lo tanto, el calibre final del fruto del palto se define en su mayor parte durante los primeros 120 días después de plena floración (Cowan et al., 2001).

Los factores climáticos, nutricionales y hormonales afectan el proceso mitótico $\mathrm{u}$ otros relacionados con el crecimiento del fruto e influencian notablemente el tamaño de la palta 'Hass'. Muchas estrategias de manejo del cultivo están orientadas a controlar tales factores o a mejorar los procesos que generan, en beneficio de un mejor calibre final (Cutting, 1993; Lovatt, 2001; Salazar-García \& Lazcano-Ferrat, 2003; Howden et al., 2005).

El anillado es una técnica que consiste en la remoción de un aro o arandela de corteza realizada por lo general en ramas. Se usa en varias especies de árboles frutales y sus efectos dependen mucho del estado fenológico de la planta o época en que se realiza. Algunas referencias indican resultados positivos del anillado en el tamaño de los frutos del palto. Así, Lahav et al. (1975) anillando árboles del cultivar Hass después del cuajado, lograron aumentar significativamente el tamaño de los frutos. Davie et al. (1995) mencionan que se muestra en promedio un aumento de masa de más de $35 \%$ en frutas de ramas del mismo cultivar anilladas en invierno. Por otro lado, Ticho (1971) con anillos de principios de otoño incrementó el calibre

\footnotetext{
${ }^{1}$ Ex- alumna. Facultad de Agronomía. Universidad Nacional Agraria La Molina, Lima, Perú. Email: sofiaflores@lamolina.edu.pe

${ }^{2}$ Universidad Nacional Agraria La Molina, Lima, Perú. Facultad de Agronomía. Email: escobedo@lamolina.edu.pe
} 
de frutos en desarrollo del palto 'Fuerte' y Díaz (1979) con el cv. Nabal anillado a fines de otoño y principios de invierno obtuvo fruta de mayor tamaño en la cosecha de esa temporada. Sin embargo, otros reportes señalan ausencia de respuesta positiva del anillado en el tamaño de los frutos, tal como lo indica Gregoriou (1989) para el cultivar Fuerte. Ramírez-Gil (2017) por su parte, reporta efectos positivos del anillado solo en el cuajado de frutos de palto pero no en producción ni aspectos de calidad.

La citoquinina parece ser la principal hormona que sustenta la actividad mitótica del fruto del palto, pues según Cowan et al. (2005), la semilla del palto 'Hass' muestra poca habilidad para producir y almacenar auxina. Algunos resultados de la aplicación de citoquininas sintéticas indican cierto sustento a esta posibilidad, como los que se reportan para la fruta del cv. Hass, que cuando es sumergida en soluciones de forclorfenuron (CPPU), a concentraciones bastante bajas que oscilan entre 2 y 10 ppm, se logra incrementar su tamaño hasta en 28 \% (Köhne, 1991; Zilkah et al., 1995). De manera similar, trabajos realizados por Ish-Am et al. (2007) confirman que aplicaciones de Benziladenina, con concentraciones menores de 100 ppm, generan efectos positivos sobre el tamaño del fruto en el cv. Ettinger. Sin embargo, Ccorimanya (2013) estudiando los calibres de la palta 'Hass' en respuesta a la carga de frutos por árbol y a la aplicación, 100 días antes de cosecha, de un producto conteniendo $10 \%$ de citoquinina sintética derivada de la adenina, solo encontró efectos de la carga de frutos y ninguna influencia del compuesto químico. En otras especies, para algunas como manzano, kiwi, vid y arándano se reportan efectos benéficos en el tamaño de los frutos de citoquininas sintéticas como thidiazuron y CPPU (Cruz et al., 1993; Vidal et al. 2003; Retamales et al., 2014); mientras que en otros frutales, como en peras, no tuvieron efecto (Dreyer, 2013).

Existe poca información sobre el efecto de las aplicaciones de $\mathrm{AG}_{3}$ en el incremento del tamaño del fruto en palto y las referencias no son muy consistentes. Así, aplicaciones de 500 ppm poco después de floración el cv. Fuerte, aumentó el número de frutos retenidos y cosechados pero no el peso de los mismos, sin embargo cuando en otro experimento se hicieron aplicaciones más tardías (al inicio de la abscisión y luego tres semanas más tarde), sin incrementarse la cosecha total se redujo el número de frutos muy pequeños y se mejoró el calibre exportable en un 17,2 \% de los frutos cosechados (Köhne, 1989; Zilkah et al., 1995). En palto 'Hass', Zhen et al., (2011), empleando $25 \mathrm{ppm}$ de $\mathrm{AG}_{3}$ aplicado en tres momentos informan de una respuesta positiva en el tamaño de los frutos solo con los tratamientos en estado de "coliflor" en el año de alta producción, mientras que la aplicación en plena floración no tuvo el efecto esperado. El hecho de que individualmente los tratamientos anteriores solo tuvieron resultados el año de alta producción sugiere que el efecto del regulador fue influenciado por la carga de frutos producidos (Lauren et al., 2011). En otras especies, como olivo, atemoya, naranjo, y aguaymanto, trabajos recientes reportan efectos positivos del ácido giberélico en el tamaño de los frutos (Faisal et al., 2012; Dos Santos et 402 al., 2016; Hifny et al., 2017; Rajesh et al., 2017).

El objetivo de la presente investigación fue evaluar el incremento del tamaño de los frutos del palto 'Hass' como respuesta del árbol a la aplicación del anillado de ramas y de dos reguladores del crecimiento (ácido giberélico y thidiazuron).

\section{Materiales y métodos}

El trabajo de investigación se realizó, entre octubre del 2011 y abril del 2012, en una plantación de 1,5 ha de árboles de palto 'Hass' de 15 años de edad injertados sobre 'Duke-7' con distanciamientos de $4 \mathrm{~m} \mathrm{x} 4 \mathrm{~m}$ y que, de acuerdo a su ciclo de alternancia productiva, se encontraban en su año de alta producción o "año on". El lote de terreno, con suelo franco arenoso homogéneo, regado por gravedad (surcos), se encuentra dentro del campus de la Universidad Nacional Agraria la Molina, Lima. El lugar corresponde a la costa central del Perú, con coordenadas $12^{\circ} 04^{\prime} 55^{\prime \prime} \mathrm{S}$ $76^{\circ} 56^{\prime} 53^{\prime \prime} \mathrm{O}$ y una altitud de 241 m.s.n.m. El experimento estuvo conformado por tres ensayos. Para cada uno de ellos se seleccionaron árboles aparentemente similares distribuidos aleatoriamente en todo el lote.

\section{Ensayo 1: Anillado de ramas}

Se estudió el efecto de dos épocas de anillado: en plena floración (26 de octubre) y 60 días después de floración (ddpf). En cada fecha, el anillado de las ramas se realizó en cinco árboles, en cada uno de los cuales fueron seleccionadas dos ramas primarias que representaban aproximadamente $1 / 3$ de la copa cada una. En una de esas ramas, a $20 \pm 2 \mathrm{~cm}$ de su base, se extrajo un anillo completo de corteza de $4 \pm 1 \mathrm{~mm}$ de grosor, constituyendo la rama anillada mientras que la otra fue considerada como rama no anillada o rama control a fin de evaluar posible efecto de la porción anillada sobre la no anillada del mismo árbol. Adicionalmente se marcaron cinco árboles testigo en los que no se practicó el anillado, pero igual se marcó una rama similar a la de los árboles anillados, Sobre las ramas primarias anilladas y no anilladas de los árboles anillados y sobre las ramas primarias marcadas de los árboles testigo, se escogió una rama secundaria de entre 4 y $5 \mathrm{~cm}$ de diámetro aproximadamente y de más de $1 \mathrm{~m}$ de largo que contenía un mínimo de 20 panículas florales. Sobre estas panículas se hicieron posteriormente las evaluaciones correspondientes.

\section{Tratamientos}

Considerando que se anilló en dos épocas diferentes, fueron cinco los tratamientos resultantes, cada uno localizado en una rama primaria como unidad experimental y con cinco repeticiones (una repetición en cada árbol). Tratamientos: T0 (Árbol testigo, sin rama anillada), T1 (Rama anillada de árbol anillado en floración), T2 (Rama no anillada de árbol anillado en floración), T3 (Rama anillada de árbol anillado 60 ddpf) y el T4 (Rama no anillada de árbol anillado 60 ddpf).

\section{Ensayo 2: Efecto de la aplicación de Thidiazurón (TDZ)} Las aplicaciones fueron foliares y se realizaron $60 \mathrm{ddpf}$ 
(26 de diciembre) con asperjador manual de 20 litros de capacidad, mojando completamente la copa de cada árbol con 5,0 litros de solución por árbol. Como fuente de la citoquinina sintética se usó el producto comercial Dropp ${ }^{\circledR}$ con $50 \%$ de pureza. Se empleó un árbol como unidad experimental con cinco repeticiones y en cada uno se marcó una rama secundaria con las características ya anotadas en el experimento anterior, de donde se tomaron los frutos para las evaluaciones. Tratamientos, Fueron cuatro los tratamientos, aplicados a cinco árboles cada uno. T0 (Testigo, sin aplicación. Se emplearon los mismos árboles testigo del ensayo 1), T1(25 ppm de TDZ), T2 (50 ppm de TDZ) y el T3 (100 ppm de TDZ).

Ensayo 3: Efecto de la aplicación del Ácido Giberélico $\left(\mathrm{AG}_{3}\right)$

Los tratamientos se aplicaron de la misma forma y fecha que las anotadas para el ensayo dos e igualmente se emplearon cinco árboles por tratamiento y se marcó la respectiva rama secundaria para la evaluación correspondiente. Tratamientos: T0 (Testigo, sin aplicación de $\mathrm{AG}_{3}$. Se emplearon los mismos árboles testigo del ensayo 1), T1 (Aplicación de 25 ppm de $\mathrm{AG}_{3}$ ) y el T2 (Aplicación de 50 ppm de $\mathrm{AG}_{3}$ ).

Diseño Experimental. En los tres ensayos, los tratamientos fueron distribuidos de acuerdo a un diseño completamente al azar (DCA). El análisis de varianza (ANVA) se realizó con los datos de diámetro y longitud del fruto, mediante el programa estadístico $\mathrm{R}$ y corroborado por Statistycal Analysis System SAS. Las diferencias entre medias se determinaron con el test HSD de Tukey con un nivel de $5 \%$.

Las evaluaciones de los tres ensayos, se llevaron a cabo el día 24 de marzo, tres semanas previas a la cosecha de los frutos. De las ramas secundarias marcadas para las evaluaciones, se tomaron 10 frutos al azar, a los cuales se les midió el diámetro ecuatorial y diámetro polar, expresado en milímetros.

\section{Resultados y discusión}

\section{Ensayo 1: Anillado de ramas}

Según los datos de la Tabla 1, con una significación estadística de $P=0,05$, en los árboles anillados en floración, el diámetro y la longitud de los frutos de la rama anillada, estadísticamente fueron similares a los de la rama no anillada del mismo árbol y a los frutos de los árboles testigo. Sin embargo, en las ramas que fueron anilladas 60 días después de plena floración, los frutos tuvieron un diámetro mayor $(60,83 \mathrm{~mm})$ que los frutos del árbol testigo $(55,59 \mathrm{~mm})$, lo que representa un incremento de 9,42\%. De la misma manera, la longitud de los frutos en las ramas con el anillado tardío $(90,44 \mathrm{~mm})$ fue superior a la de los frutos sobre los árboles testigo $\mathbf{( 7 9 , 9 9} \mathbf{~ m m})$, que implica un 13,06 \% de aumento en la longitud. Además, la rama anillada no afectó las medidas de los frutos de la rama no anillada del mismo árbol pues esta produjo frutos con el mismo diámetro y longitud que los árboles testigo.

De acuerdo con lo manifestado por Whiley et al. (2013), la interrupción temporal del flujo descendente por el floema, debió de crear en la rama anillada una situación especial particularmente favorable por la acumulación de substancias de naturaleza variada que se redistribuyeron por sobre el anillado, beneficiando de esta manera, los procesos que ocurran mientras el anillo permaneció operativo. El anillado tardío empezó su efecto cuando ya prácticamente todos los frutos habían iniciado su crecimiento, y lo prolongó todo el tiempo que demoró en cicatrizar (por lo menos 60 a 80 días más tarde), es decir que estuvo activo en la etapa más sensible de la división celular después del cuajado que según Cowan et al. (2001), se prolonga hasta 55 a 60 días después de este. El anillado en floración aparentemente orientó los recursos a procesos más tempranos relacionados con el cuajado de los frutos pues según Tomer (1977), cuando se realiza en esa época, se promueve el incremento de la longitud del tubo polínico y su penetración al interior del óvulo. El efecto positivo en el tamaño de los frutos que se alcanzó con el anillado realizado 60 días después de plena floración, y cuyos resultados estuvieron limitados solo a la campaña en que se realizó el estudio (año “on”), concuerda con lo reportado por otros investigadores quienes, en palto 'Hass' y otros cultivares, encontraron respuesta similar con anillados tardíos, de otoño o principios de invierno (Ticho, 1971; Díaz, 1979; Davie et al., 1995).

Tabla 1. Diámetro y longitud de frutos $(\mathrm{mm})$, en árboles anillados en floración y 60 días después de plena floración

\begin{tabular}{lccc}
\hline & Tratamiento & $\begin{array}{c}\text { Diámetro de } \\
\text { fruto }(\mathrm{mm})\end{array}$ & $\begin{array}{c}\text { Longitud de } \\
\text { fruto }(\mathrm{mm})\end{array}$ \\
\hline Árbol sin anillar & Árbol testigo & $55,59 \mathrm{~b} *$ & $79,99 \mathrm{~b}$ \\
Árbol anillado & Rama no anillada & $55,81 \mathrm{~b}$ & $81,53 \mathrm{~b}$ \\
En floración & Rama Anillada & $58,31 \mathrm{ab}$ & $83,97 \mathrm{~b}$ \\
$\begin{array}{l}\text { Árbol anillado 60 } \\
\text { días después de }\end{array}$ & Rama no anillada & $56,53 \mathrm{~b}$ & $85,10 \mathrm{ab}$ \\
plena floración & Rama Anillada & $60,83 \mathrm{a}$ & $90,44 \mathrm{a}$ \\
\multicolumn{2}{c}{ Diferencia mínima significativa } & 2,890 & 6,309 \\
\hline
\end{tabular}

*En cada columna, letras iguales indican que los tratamientos no difieren estadísticamente según el test HSD de Tukey con $P=0.05$

\section{Ensayo 2: Aplicación de Thidiazurón (TDZ)}

Como se observa en la Tabla 2, a un nivel de significación estadística de $\mathrm{P}=0,05$, se registraron frutos con diámetros mayores al testigo $(55,59 \mathrm{~mm})$ solo con las dosis más bajas de TDZ: $60,10 \mathrm{~mm}$ para $25 \mathrm{ppm}(8,11 \%$ de incremento) y $61,04 \mathrm{~mm}(9,80 \%$ de incremento) para $50 \mathrm{ppm}$ y $\sin$ diferencias entre ambas. Sin embargo, la longitud de los frutos fue mejor en los árboles que recibieron las tres concentraciones del producto: $86,64 \mathrm{~mm}, 85,90 \mathrm{~mm}$ y $86,63 \mathrm{~mm}$ para 25,50 y $100 \mathrm{ppm}$ respectivamente, y sin diferencias entre las tres dosis, en tanto que los frutos de los testigos alcanzaron únicamente 79,99 $\mathrm{mm}$ de longitud, lo que en porcentaje representa $8,31 \% ; 7,38 \%$ y $8,30 \%$ de aumento en la longitud de los frutos para cada una de las concentraciones anotadas.

Resultados positivos en el calibre de los frutos encontraron Ish-Am et al. (2007) en frutos de palto 'Ettinger' con otra citoquinina sintética, la Benziladenina, a concentraciones menores a $100 \mathrm{ppm}$. Es posible que estos 
efectos tengan relación con el incremento del poder de sumidero de los frutos como parecen sugerir los resultados reportados por Nardozza et al. (2017), quienes encontraron que las aplicaciones de la citoquinina sintética CPPU aumentaron el peso de los frutos de kiwi por incremento de la demanda de carbohidratos y al haber limitación en su abastecimiento, absorben más agua. También es posible que la citoquinina participe como agente de activación en la intensificación de las divisiones celulares, asociadas con los estados iniciales del crecimiento del fruto (Taiz y Zeiger, 2006),

Tabla 2. Diámetro y longitud ( $\mathrm{mm})$ de los frutos, en árboles tratados 60 ddpf con TDZ

\begin{tabular}{ccc}
\hline $\begin{array}{c}\text { Tratamiento } \\
\text { TDZ }(\mathrm{ppm})\end{array}$ & $\begin{array}{c}\text { Diámetro de } \\
\text { fruto }(\mathrm{mm})\end{array}$ & $\begin{array}{c}\text { Longitud de } \\
\text { fruto }(\mathrm{mm})\end{array}$ \\
\hline 0 (Testigo) & $55,59 \mathrm{c} *$ & $79,99 \mathrm{~b}$ \\
$25 \mathrm{ppm}$ & $60,10 \mathrm{ab}$ & $88,64 \mathrm{a}$ \\
$50 \mathrm{ppm}$ & $61,04 \mathrm{a}$ & $85,90 \mathrm{a}$ \\
$100 \mathrm{ppm}$ & $57,79 \mathrm{bc}$ & $86,63 \mathrm{a}$ \\
Diferencia mínima significativa & 2,323 & 4,700 \\
\hline *En cada columna, letras iguales indican que los tratamientos no difieren \\
estadísticamente según el test HSD de Tukey con $P=0.05$
\end{tabular}

Ensayo 3: Efecto de la aplicación de ácido giberélico $\left(\mathrm{AG}_{3}\right)$

De forma general, a un nivel de significación estadística de $\mathrm{P}=0,05$, los diámetros y longitudes de los frutos no mejoraron con los tratamientos y fueron iguales a los registrados para los árboles testigo, según se observa en la Tabla 3.

Tabla 3. Diámetro y longitud ( $\mathrm{mm})$ de los frutos, en árboles tratados 60 ddpf, con AG3

\begin{tabular}{lccc}
\hline & $\begin{array}{c}\text { Tratamiento } \\
\mathrm{AG}_{3}(\mathrm{ppm})\end{array}$ & $\begin{array}{c}\text { Diámetro de } \\
\text { fruto }(\mathrm{mm})\end{array}$ & $\begin{array}{c}\text { Longitud de } \\
\text { fruto }(\mathrm{mm})\end{array}$ \\
\hline 0 (Testigo) & $55,59 \mathrm{a}^{*}$ & $79,99 \mathrm{a}$ \\
$25 \mathrm{ppm}$ & $59,03 \mathrm{a}$ & $83,18 \mathrm{a}$ \\
$50 \mathrm{ppm}$ & $57,95 \mathrm{a}$ & $83,12 \mathrm{a}$ \\
Diferencia mínima significativa & 3,753 & 5,520 \\
\hline
\end{tabular}

*En cada columna, letras iguales indican que los tratamientos no difieren estadísticamente según el test HSD de Tukey con $P=0.05$

Los resultados inconsistentes y faltos de uniformidad con aplicaciones de $\mathrm{AG}_{3}$ para incrementar el tamaño de los frutos, son frecuentes y están sujetos, al parecer, a factores muy específicos de las plantas y del medio ambiente. Algunas investigaciones indican que este regulador del crecimiento empleado en palto 'Hass' a concentraciones de $25 \mathrm{ppm}$ incrementa el calibre de los frutos cuando se aplica entre estado de "coliflor" y cuatro meses más tarde (marzo y julio en el hemisferio norte) solo en el año "on" (Lovatt y Salazar-García, 2003; Lovatt, 2005). Posiblemente la aplicación de la giberelina se realizó en época muy tardía o la carga de frutos de los árboles experimentales, a pesar de estar en su año "on" no fue muy alta y esto pudo afectar los resultados, tal como lo sugiere también Lauren et al. (2011).

De manera general, los resultados de los tres ensayos indican que el anillado de inicios de verano y la aplicación 404 de dosis bajas de TDZ (25-50 ppm) deberían considerarse como una posibilidad en el manejo de plantaciones de palto 'Hass' con problemas de bajo calibre de fruta.

\section{Conclusiones}

El anillado de las ramas fue efectivo cuando se realizó 60 días después de plena floración, incrementando el diámetro y la longitud de los frutos. Dosis de 25 y 50 y 100 ppm de TDZ aplicadas en la época mencionada anteriormente también resultaron beneficiosas. Las tres incrementaron la longitud de los frutos, mientras que el diámetro solo resultó mejor con las dos dosis más bajas. La aplicación de $\mathrm{AG}_{3}$ no tuvo efectos positivos en el incremento del tamaño del fruto.

\section{Literatura Citada}

Bergh, B. 1984. Avocado Varieties for California. Calif. Avocado Soc. Yrbk., 68:75-94.

Ccorimanya, R. 2013. Aplicación de citoquinina (Citogrower ${ }^{\circledR}$ ) y raleo de frutos para su respuesta en la productividad del cultivo de palto (Persea americana Mill. cv. Hass) bajo condiciones de la irrigación Majes. Tesis Ing. Agr. Universidad Nacional de San Agustín de Arequipa, Perú.

Cowan, A.; Crips, R.; Richings, E. and Taylor, N. 2001. Fruit size: Towards an understanding of the metabolic control of fruit growth using avocado as a model system. Physiol. Plant., 111: 127-136.

Cowan, A.; Taylor, N. and Van Staden, J. 2005. Hormone homeostasis and induction of the small-fruit phenotype in 'Hass' avocado. Plant Growth Regulation, 45: 11-19.

Cruz, J.; Woolley, J. and Lawes, S. 1993. The effects of seeds and the application of a growth regulator mixture, on fruit growth in 'Hayward' kiwifruit. Acta Horticulturae 329: 128-130.

Cutting, J. 1993. The cytokinin complex as related to small fruit in 'Hass' avocado. South African Avocado Growers' Association Yearbook, 16:20-21 .

Davie, S., Stassen, P. and Van der Walt, M. 1995. Girdling for increased 'Hass' fruit size and its effect on carbohydrate production and storage. Proceeding of World Avocado Congress III. Tel Aviv. Israel. 33-36p.

Diaz, M. 1979. Anillado en paltos en la variedad Nabal. Tesis Ing. Agrónomo. Pontificia Universidad Católica de Chile, Facultad de Agronomía. Chile.

Dos Santos, R.; Pereira, M.; Mendes, D.; Soares, R.; Nietsche, S.; Mizobutsi, G.; Dos Santos, B. 2016. Gibberellic acid induces parthenocarpy and increases fruit size in the 'Gefner' custard apple (Annona cherimola $x$ Annona squamosa). Australian Journal of Crop Science, 10(3): 314-321.

Dreyer, C. 2013. Fruit set and fruit size studies on 'Forelle' and 'Abate Fetel' pear (Pyrus communis L.). Thesis presented in partial fulfilment of the requirements for the degree Master of Science in Agriculture (Horticultural Science) in the Faculty of AgriSciences, 
at Stellenbosch University. South Africa.

Faisal, K.; Khalid, M.; Ammaz, K.; Fakhar-Ul, H. and Nabila, B. 2012. Effect of girdling and plant growth regulators on productivity in olive (Olea europaea). Pakistan J. Agric. Res., 25 (2): 120-128.

Gregoriou, C. 1989. Effect of girdling on fruit set of Fuerte avocado variety. Calif. Avocado Soc. Yrbk., 73:153158.

Hifny, H.; Khalifa, S.; Hamdy, A.; Wahed, 2017. Effect of GA3 and NAA on growth, yield and fruit quality of Washington Navel orange. Egypt. J. Hort., 44(1): 33 43.

Howden, M.; Newett, S. and Deuter, P. 2005. Climate change -risks and opportunities for the avocado industry. New Zealand and Australia Avocado Grower's Conference '05. 20-22 September 2005. Tauranga, New Zealand. Session 1. Introduction, $19 \mathrm{p}$.

Ish-Am, G.; Cohen, H.; Eidelman, E.; Regev, I; Winer, L. and Lahav, E. 2007. Achieving larger 'Ettinger' fruits in western Galilee, Israel. Proceeding VI World Avocado Congress 2007. Viña del Mar, Chile. 12-16.

Jaque, R. 2006. Efecto del calibre y la altura del fruto dentro del árbol sobre el contenido de aceite, en palta (Persea americana Mill.) cv. Hass y Fuerte. Tesis. Pontificia Universidad Católica de Valparaíso. Chile.

Köhne, J. 1989. Spring growth of avocado trees as influenced by pruning and the use of growth regulators to stabilize yield and improve fruit quality. Hohenheim University, Stuttgart, $\mathrm{PhD}$ thesis.

Köhne, J. 1991. Increasing 'Hass' fruit size. Proceedings of the Second World Avocado Congress: 21 - 26. April 1991. Orange, California, USA.

Lahav, E.; Gefen, B. and Zamet, D. 1975. Increasing the size of Hass avocado fruits. Scientific Activities 19711974. Institute of Hort., Bet Dagan. Israel.

Lauren, G.; Klein, G.; Zheng, Y.; Khuong, T. and Lovatt, C. 2011. Response of evergreen perennial tree crops to gibberellic acid is crop load-dependent: II. GA3 increases yield and fruit size of 'Hass' avocado only in the on-crop year an alternate bearing orchard. Scientia Horticulturae, 130(4): 753-761.

Lovatt, C. 2001. Properly timed soil-applied nitrogen fertilizer increases yield and fruit size of 'Hass' avocado. J. Amer. Soc. Hort. Sci. 126(5):555-559.

Lovatt, C. 2005. Plant growth regulators for avocado production. Calif. Avocado Soc. Yrbk., 88: 81-91

Lovatt, C. y Salazar-García, S. 2003. La aplicación foliar de ácido giberélico $\left(\mathrm{AG}_{3}\right)$ en momento adecuado aumenta el tamaño del fruto y el rendimiento durante el año de alta producción, incrementando el rendimiento acumulado. V Congreso Mundial del Aguacate. Resúmenes. A-108. 266-267p.

Moore-Gordon, C.; Cowan, A.; Bertling, I.; Botha, J. and Cross, R. 1998. Symplastic solute transport and avocado fruit development: a decline in cytokinin/ ABA ratio is related to appearance of the 'Hass' small fruit variant. Plant Cell Physiol., 39 (10): 1027-1038.

Nardozza, S.; Boldingh, H.; Wohlers, M.; Gleave, A.; Luo. Z.; Costa, G.; MacRae, E.; Clearwater, M. and Richardson, A. 2017. Exogenous cytokinins application to Actinidia chinensis var. deliciosa 'Hayward' promotes fruit expansion through water uptake. Horticultural Research 4, Article number: 17043.

Rajesh, K.; Singh, S.; Anupam, T.; Sutanu, M.; Vishnu L. 2017. Effect of gibberellic acid (GA3) on fruit yield and quality of cape gooseberry (Physalis peruviana L.). International Journal of Advanced Biological Research, 7(4): 724-727

Ramirez-Gil, J. 2017. Calidad del fruto de aguacate con aplicaciones de ANA, boro, nitrógeno, sacarosa y anillado. Agron. Mesoam., 28(3): 591-603

Retamales, J.; Lobos, G.; Romero, S; Godoy, R.; Moggia, C. 2014. Repeated applications of CPPU on highbush blueberry cv. Duke increase yield and enhance fruit quality at harvest and during postharvest. Chilean J. Agric. Res., 74(2): 157-161

Salazar-García, S.; Lazcano-Ferrat, I. 2003. Site-specific fertilization increased yield and fruit size in 'Hass' avocado. Better Crops International 17(1): 12-15.

Taiz, L.; Zeiger, E. 2006. Fisiología vegetal. $3^{\circ}$ Edición. USA. pp. 913-914.

Ticho, R. 1971. Girdling, a means to increase avocado fruit production. Calif. Avocado Soc. Yrbk., 54:90-94.

Tomer, E. 1977. The Effect of girdling on flowering, fruit setting and abscission in avocado trees. The Hebrew Univ. of Jerusalem, Israel, unpublished PhD thesis. 138 p. En Berrios, M. 1994. Efecto del anillado, doble incisión anular y aplicaciones de paclobutrazol (cultar) en paltos (Persea americana Mill.) cv. Negra de la Cruz. Tesis Ingeniero Agrónomo. Univ. Católica de Valparaíso. Chile.

Vidal, C.; Aparecida, C.; Bassay, L. 2003. Effect of preharvest spraying with thidiazuron on fruit quality and maturity of apples. Rev. Bras. Frutic., Jaboticabal - Sp. 25(1): 59-62.

Whiley, A.; Wolstenholme, B.; Faber. B. 2013. Manejo del cultivo, pp. 405-448. In: Schaffer, B.; Wolstenholme, B; Whiley, A. El aguacate: Botánica, producción y usos. 2 ed. Chile: CABI, Ediciones Universitarias de Valparaiso, Chile.

Zhen, Y; Khuong, T.; Lovatt, C. 2011. Effect of foliar applied plant bio regulators on 'Hass' avocado yield. Proceedings VII World Avocado Congress 2011. Cairns, Australia. 5-9 sept.

Zilkah, S.; David, I.; Yeselson, Y.; Tamir, M.; Winer, L. 1995. Increasing 'Hass' avocado fruit size by CPPU and GA application. Proceedings of the III World Avocado Congress. Israel.

Zilkah, S. and Klein I. 1987. Growth kinetics and determination of shape and size of small and large avocado fruits cultivar Hass on the tree. Scientia Horticulturae, 32: 195 - 202. 